\title{
3D chaos graph deep learning method to encrypt and decrypt digital image
}

\author{
Daniah Abdul Qahar Shakir, Ali Jbaeer Dawood \\ University of Anbar, College of Computer Science and Information Technology, Ramadi, Iraq
}

\begin{abstract}
Article Info
Article history:

Received Aug 8, 2021

Revised Dec 1, 2021

Accepted Dec 15, 2021

\section{Keywords: \\ 3D chaos map \\ Decryption \\ Deep learning \\ Digital images \\ Encryption \\ Multimedia}

ABSTRACT

We live in technological age development's where many important data transmitted electronically from one device to another and in every place. Deep learning algorithms have facilitated the process of encoding and decoding digital images. Chaotic graph systems, on the other hand, are one of the most recent techniques utilized to encode image data based on the methods of cryptography. The chaos maps are divided into two main aspects, first one deals with the 1D map which requires fewer features and can be developed easily, the second one is the high dimensional map which is more complex than the $1 \mathrm{D}$ graph and it requires more features, more parameters, and it is relatively hard to develop. In this paper, we present a method for image encoding and decoding electronically using deep learning, the proposed algorithm was developed by using the hybrid technique of $3 \mathrm{D}$ chaos map generation, the best case of the proposed technique gave the following results: The average entropy calculation was (7.4838) before image encryption and (7.9896) after image encryption with average number of pixels change rate (NPCR) of $(99.7085 \%)$ and the unified average changing intensity (UACI) of (33.2030\%) which are the best outcomes when compared to other similar works.
\end{abstract}

This is an open access article under the CC BY-SA license.

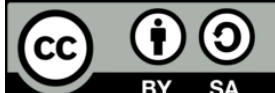

\section{Corresponding Author:}

Daniah Abdul Qahar Shakir

Department of Computer Science, University of Anbar

Anbar, Iraq

Email: dan14c1001@uoanbar.edu.iq

\section{INTRODUCTION}

Securing data communication became a must in the digital era, cryptography is the perfect method to remain the safety of the transmitted data over the communication channels, this field must ensure the four principles of cryptography namely: data confidentiality, integrity, authentication, and data non-repudiation [1]. Various algorithms have been taken into the place of date encryption and decryption specially to protect the multimedia data since these data are the most usable data across the internet [2]. Digital image encoding/decoding methods are widely examined rather than the traditional digital data cryptography methods such as the traditional data encryption standard (DES) algorithms which may not be able to suit the large-scale, highly complex digital images [3].

Machine learning algorithms vary depending on the type and features of the data along with the situation where how the research problem and aim were designed [4]. Data mining and deep learning tools have been given a promising outcome in both classification and clustering learning techniques especially when it comes to large-scale, complex, high dimensional data such as the image encryption and decryption method [5]. The aspect of image security thrives with artificial neural networks that confirm the data confidentiality and accuracy, but it is an expensive tool to implement with complex and sensitive data such as the image [6]. Images carry out huge information which makes it challenging to perform the 
encoding/decoding algorithms as the original text-based dataset. On the other hand, the image resolution and quality after the decoding process must be unchangeable which a quite challenging task [7], [8].

This paper proposes a modern digital image cryptography method with a chaotic map based on deep learning for an advanced, highly secure, and robust technique. This method ensures that images can be sent safely without worrying about information leakage. Recent works have examined digital image security and proposed different methods to protect the data of the images [9].

- Chen et al. [10] implemented artificial neural nets with impulse synchronization technique with nonlinear pulse controller, then developed this modern method to a digital image which showed a highly secure outcome.

- $\quad$ Dridi et al. [11] designed a new artificial neural network (ANN) approach that lessens the complexity of the digital medical image cryptography, pixel summation, and exclusive or (XOR) operation was used to ensure the performance of the method which confirms the effectiveness and the robustness of the neural networks.

- Fakhr [12] developed modern compression of key sensing technology with machine learning, it computes the square of the Euclidean distance long with the dot product then test the results using COREL image.

- Daolin et al. [13] utilized CryptoNets which are the cryptography with ANN was utilized cloud computing to ensure the safety of the transmitted image, this technique showed a divine result when tested using the modified national institute of standards and technology (MNEST) dataset.

- Hu et al. [14] implanted a network called the stack automatic encoder (SAE) to shuffle the coordinates of the pixels into the image which enhanced the encryption process and it showed that this method is robust against brute force attack, differential attack, and statistical attack.

- $\quad$ Hu et al. [15] steganography method was produced to enhance the image encoding, carrier image was developed using ANN to ensure that the data remains the same with no update or alter to its information with highly accurate and robust information extraction.

- Lu et al. [16] the equations of the second order were switched to the first order with LyapunovKrasovskii functional and Jensen's inequality functions along with chaotic ANN method was developed for reliable and efficient image transformation.

- Shifa et al. [17] produced advanced encryption standard (AES) technology with the 3 channel images i.e., the colored images rather than changing the image to grayscale first, this contribution has shown ideal information hiding with accurate performance.

- $\quad$ Liu et al. [18] chaotic S-Box, logistic-sine system (LSS) schema, and chaotic key were produced in this work which makes the method resistant against the certified public accounts (CPA) and it works perfectly with the real-time encoding of the images.

- $\quad$ Rungruanganukul and Siriborvornratanakul [19] proposed building and training a convolutional neural network from scratch using Google Colab's deep learning framework. The trained network is part of the core artificial intelligence of interactive software games, aimed at encouraging employees to exercise their hands and wrists frequently during the game. The network is trained on a self-collecting dataset of 12,000 images recorded against a static dark background, using simple gestures. The network focuses on classifying still images into one of six predefined gesture classes, handling small changes in size, skin color, position, and hand orientation. This network was designed to be easily calculated with realtime runtime even on the central processing unit (CPU). The network provides 99.68 accuracy in the validation set, with an average accuracy of $78 \%$ when tested with 50 different users.

\section{METHODOLOGY}

Explaining the proposed research methodology will be in detail in this section. Research design and research procedure (in the form Pseudocode) will be illustrated as presented in the following flowchart where the proposed work generated a 3D chaotic map from the concept that originated from the 2D chaotic map of image encryption. After that the original images will be fed one by one to the proposed system to encrypt and decrypt the results, along the process histogram equalization will be implemented which transforms the 3D Image channels to 2D (grey scale). Then applying the image rotation and XOR equations. Finally, hybrid image encryption-decryption technique will be produced. Figure 1 illustrates the entire process, 


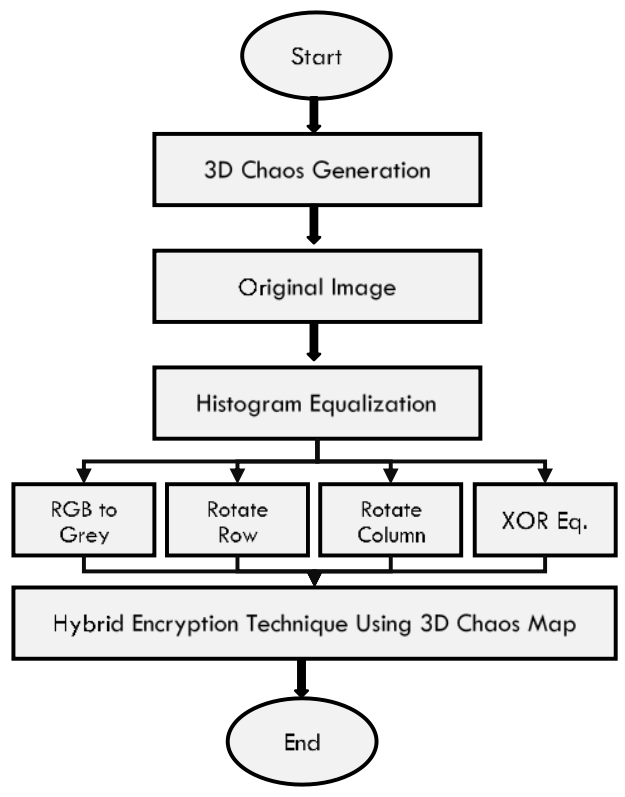

Figure 1.3D chaotic map with image encryption implantation

\subsection{Key generation}

1. 3D logistic map is used to produce the behavior of the chaotic map, to reach a 3D chaotic map, 2D Map will be he start point, 2D map was proposed by Liu et al. as a 2D logistic map given by the following equations [20],

$$
\begin{aligned}
& X_{i+1}=\mu_{1} X_{i}\left(1-x_{i}\right)+y_{1}^{1} y_{i}^{2} \\
& Y_{i+1}=\mu_{2} Y_{i}\left(1-y_{i}\right)+y_{2}^{1}\left(x_{i}^{2}+x_{i} y_{i}\right)
\end{aligned}
$$

in this work, 3D key generation consists of three main parameters of the key which are (x, $\mathrm{y}$, and $\mathrm{z}$ ) along with three symbols $(\mu, \beta$, and $\gamma$ ) where the values of the mentioned symbols should be in a range from [0 to 1]. The values of each variable is given in Table 1 .

The equations on which the chaotic actions were given to the proposed method is illustrated in (3), (4), and (5) [21],

$$
\begin{aligned}
& X_{n+1}=\mu\left(1-x_{n}\right)+\beta y_{n}^{2} x_{n}+\gamma z_{n}^{3} \\
& Y_{n+1}=\mu\left(1-y_{n}\right)+\beta z_{n}^{2} x_{n}+\gamma x_{n}^{3} \\
& Z_{n+1}=\mu\left(1-z_{n}\right)+\beta x_{n}^{2} x_{n}+\gamma y_{n}^{3}
\end{aligned}
$$

Table 1. Variables range values

\begin{tabular}{ccc}
\hline Symbol & $>$ & $<$ \\
\hline$\mu$ & 3.35 & 3.81 \\
$\mathrm{~B}$ & 0 & 0.022 \\
$\Gamma$ & 0 & 0.015 \\
\hline
\end{tabular}

2. To produce the histogram of the key, an equalization process is given to the grayscale of the original image, where the image has the row and column with $\mathrm{A} * \mathrm{~B}$ dimension only the histogram equalization is given to the variables of the key (x), (y), and (z) as shown in questions (6), (7), and (8),

$$
\begin{aligned}
& X=(\text { integer }(x * i)) \bmod A \\
& Y=(\text { integer }(y * i)) \bmod B \\
& Z=(\text { integer }(x * i)) \bmod 256
\end{aligned}
$$


where integer values are always set to be greater than 10000 , where A and B are equal to 256 .

3. Column and Row Rotation: to change the position of the image pixels, it is known that the image is transformed to the grayscale e level and the dimensionality is limited to 2D only with A and B symbols each presents the row and the column. A random number (i) was generated when $i 1=1000$ rotate the row according to (7), and when the random number $i 3=1000$ rotate the column according to (8) with both respect the values of $\left(x^{\prime}\right)$ and $\left(y^{\prime}\right)$. In both cases, rotate to the right when chaos is even, otherwise rotating to the left.

4. XOR operation is implanted to the values of the pixels sequence, where the values remain hidden to the attacker unless he/she has the chaotic key, the XOR starts from i5 values after that the image dimension $(\mathrm{A} * \mathrm{~B})$ shuffled to produce the encrypted image.

\subsection{Pseducode}

Pseudocode is proposed to implement the work of this paper. It consists of 18 steps. The pseudocode of the overall work is illustrated in the following points:

Start:

Step 1: Set the initial values: key, chaotic behavior symbols.

Step 2: Define the output: encrypted image.

Step 3: Define the input images.

Step 4: Change the 3D image input to $2 D$ image input.

Step 5: Design the image height.

Step 6: Set the encryption phase.

Step 7: Prepare the equations of the initial key conditions.

Step 8: Set the 3D chaotic values to the variables and the symbolic values.

Step 9: Start a for loop to all the random number (i) values.

Step 10: Develop the histogram equalization phase.

Step 11: Set of conditions for row and column rotation.

Step 12: Reshape the image with XOR operation implantation.

Step 13: Start the decryption phase.

Step 14: Set of conditions for row and column rotation.

Step 15: Calculate the entropy.

Step 16: Calculate the coefficient correlation.

Step 17: Calculate number of pixels change rate (NPCR) and unified averaged changed intensity (UACI).

Step 18: Print the outputs.

End

\section{Experimantal Results}

In this section six main experemntial results will be given in the following tables, in is noted that three different images were utilized during the statistical analysis of these images, matrix laboratory (MATLAB) software was utilized to run the results, the outcomes will be shown in the following sections:

1. Image pixels diffusion results: three images where given (owl, birds, and buterfliy) the original 3D channels of these images are switched to 2D greyscale then their pixels are diffused, as shown Table 2.

2. Histogram Visulazation: from the visulzation of the encrypted image histogram it is visula that the values are distributed over the resultd image evenly which indicates that there is no useful information that can be extracted from the encrypted image, as shown in Tables 3, 4, and 5.

3. The calculation of the entropy: the besst case of the entroy calculation theroitcally is 8 values, the higher the inforamtion entropy embedded into the image the higher uneven values distribution of the results, the entropy was applied to the three images and the entropy of both the original and the encoded image were reistered as mentioned in Table 6.

4. The calculation of the correlation coffiecent: the correlation coefficients between vertically adjacent pixels and horizontally adjacent pixels of an encrypted image are given in Tables 7, 8, and 9 .

5. The calculation of NPCR and UACI: the former means that it is the number of changing pixel rate. (NPSCR) That gives tha avarage changed pixels in the encoded image, while the latter indicates that it is the averaged changed intensity (UACI) that gives the avarage different of the encrypted image with respect to its dimentions $\mathrm{A} * \mathrm{~B}$, the results were examined with the owl image only and it is mentioned in Table 10.

6. Comparision with previous works: this comparision was made with respect to both encryption and sensitiveity analysis with owl image which is the proosed image with our work and with different images from other similr works in the field of image encryption, Tables 11 and 12 show the analysis results. 
Table 2. Pixels diffusion results

\begin{tabular}{l} 
Original Image 3D \\
\hline
\end{tabular}

Table 3. Histogram visualization of the $1^{\text {st }}$ image

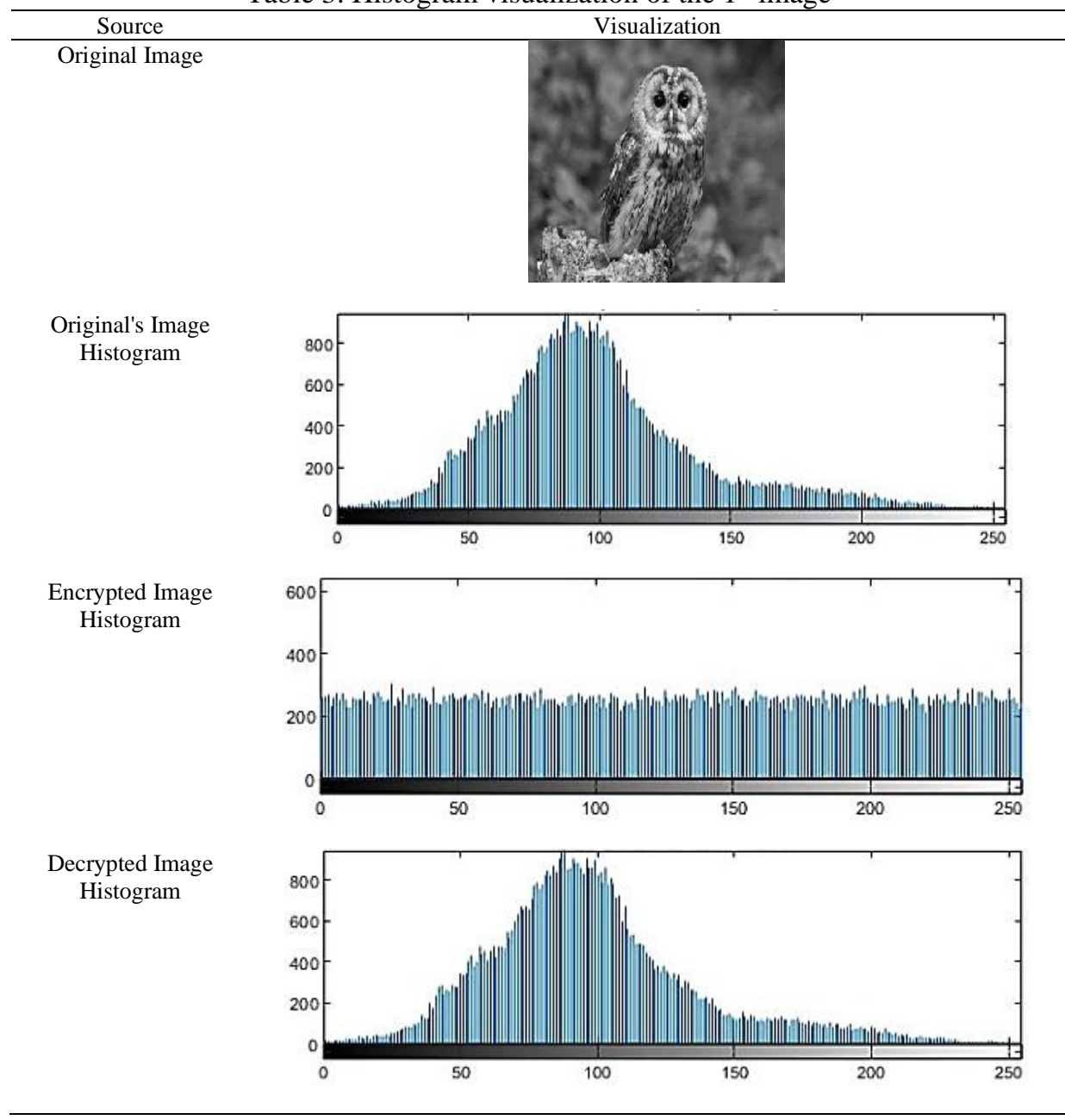

3D chaos graph deep learning method to encrypt and decrypt digital image (Daniah Abdul Qahar Shakir) 
Table 4. Histogram visualization of the $2^{\text {nd }}$ image

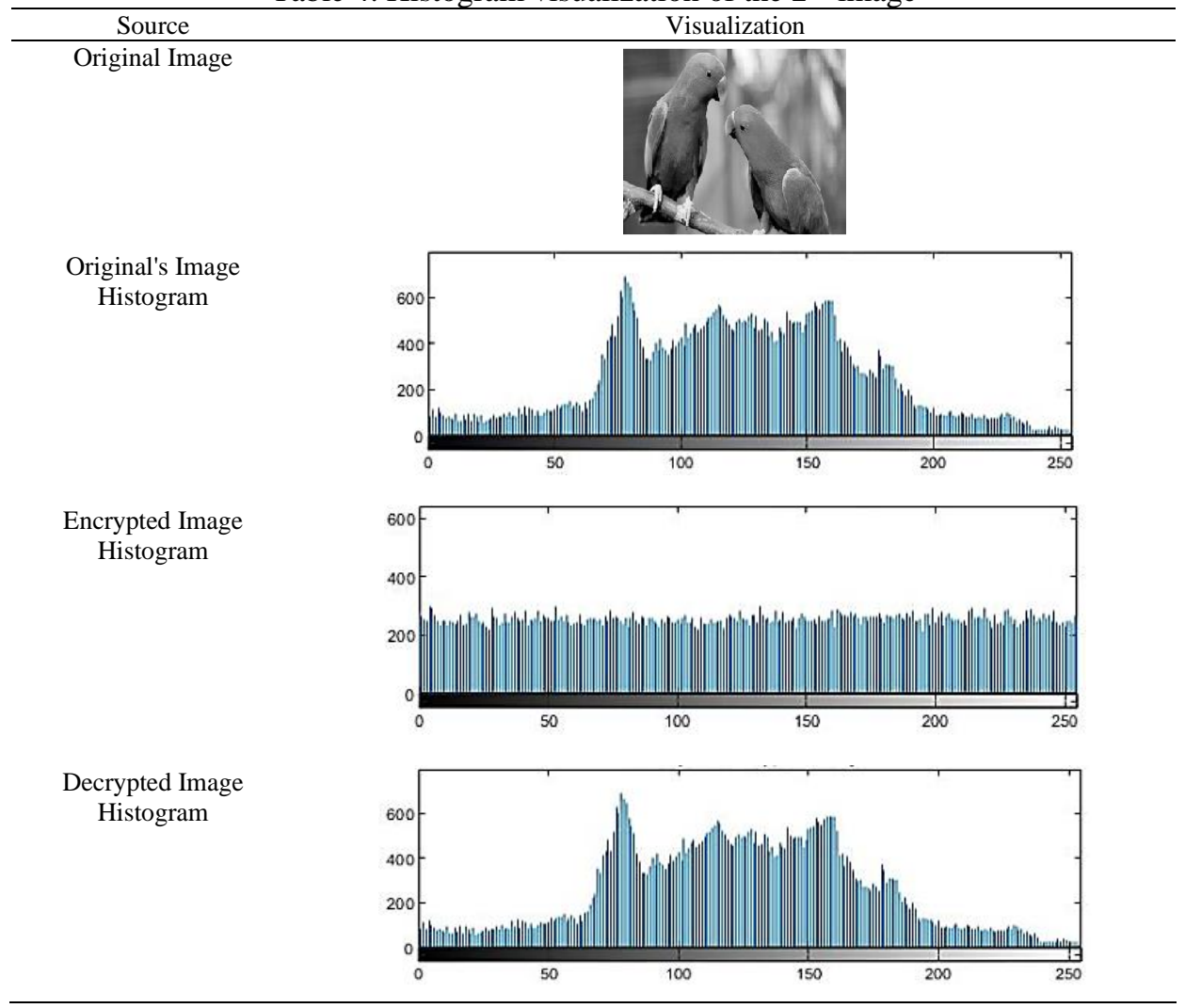

Table 5. Histogram visualization of the 3rd image

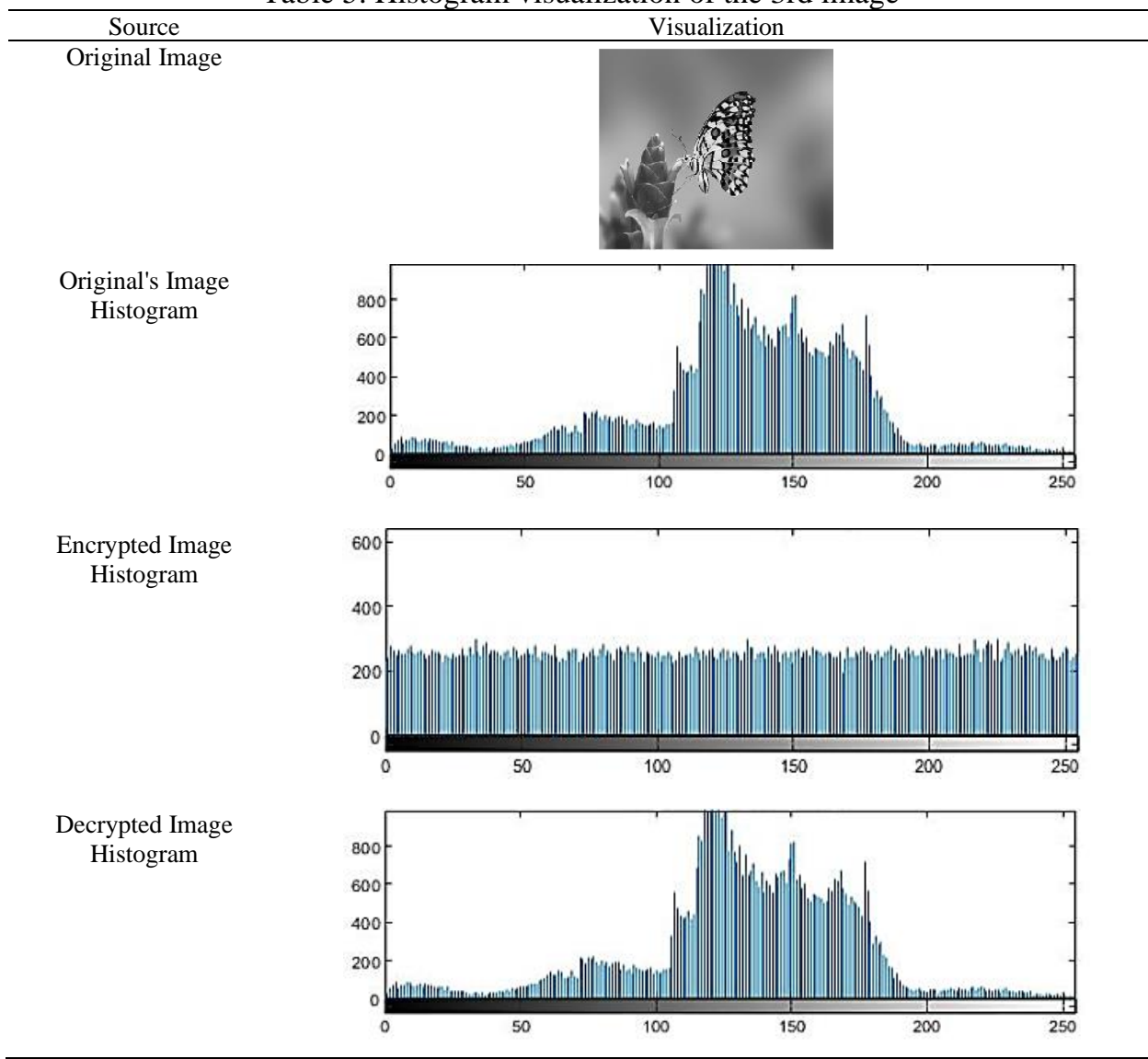

Indonesian J Elec Eng \& Comp Sci, Vol. 25, No. 2, February 2022: 941-951 
Table 6. Entropy calculation of the implented imges

\begin{tabular}{lcc}
\hline & Original Entropy & Encrypted Entropy \\
\hline OW1 Image & 7.4838 & 7.9896 \\
Birds Image & 7.2543 & 7.9887 \\
Butterflies Image & 7.6333 & 7.9894 \\
\hline
\end{tabular}

Table 7. Correlation coefficient calculation of owl image

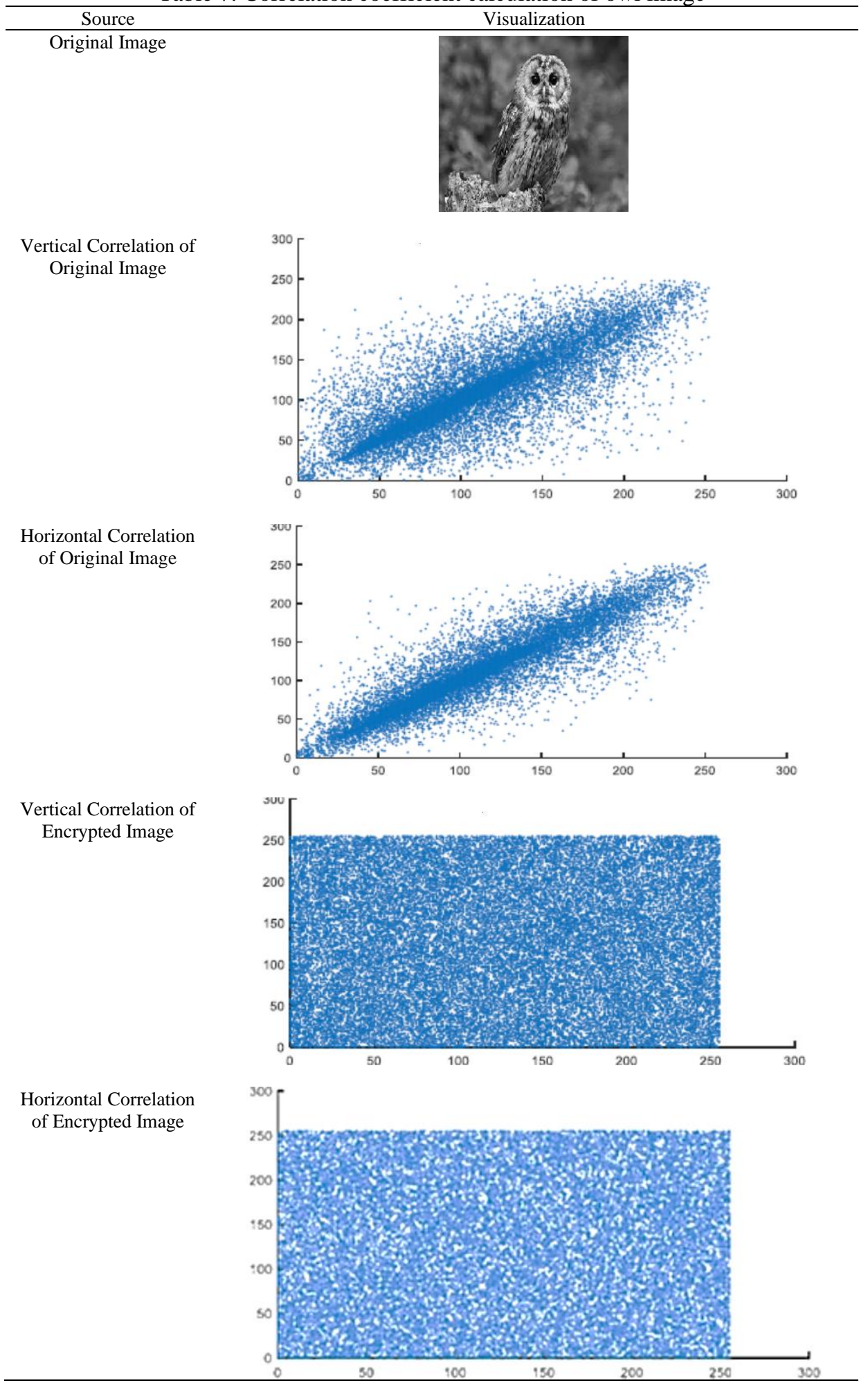


Table 8. Correlation coefficient calculation of birds image

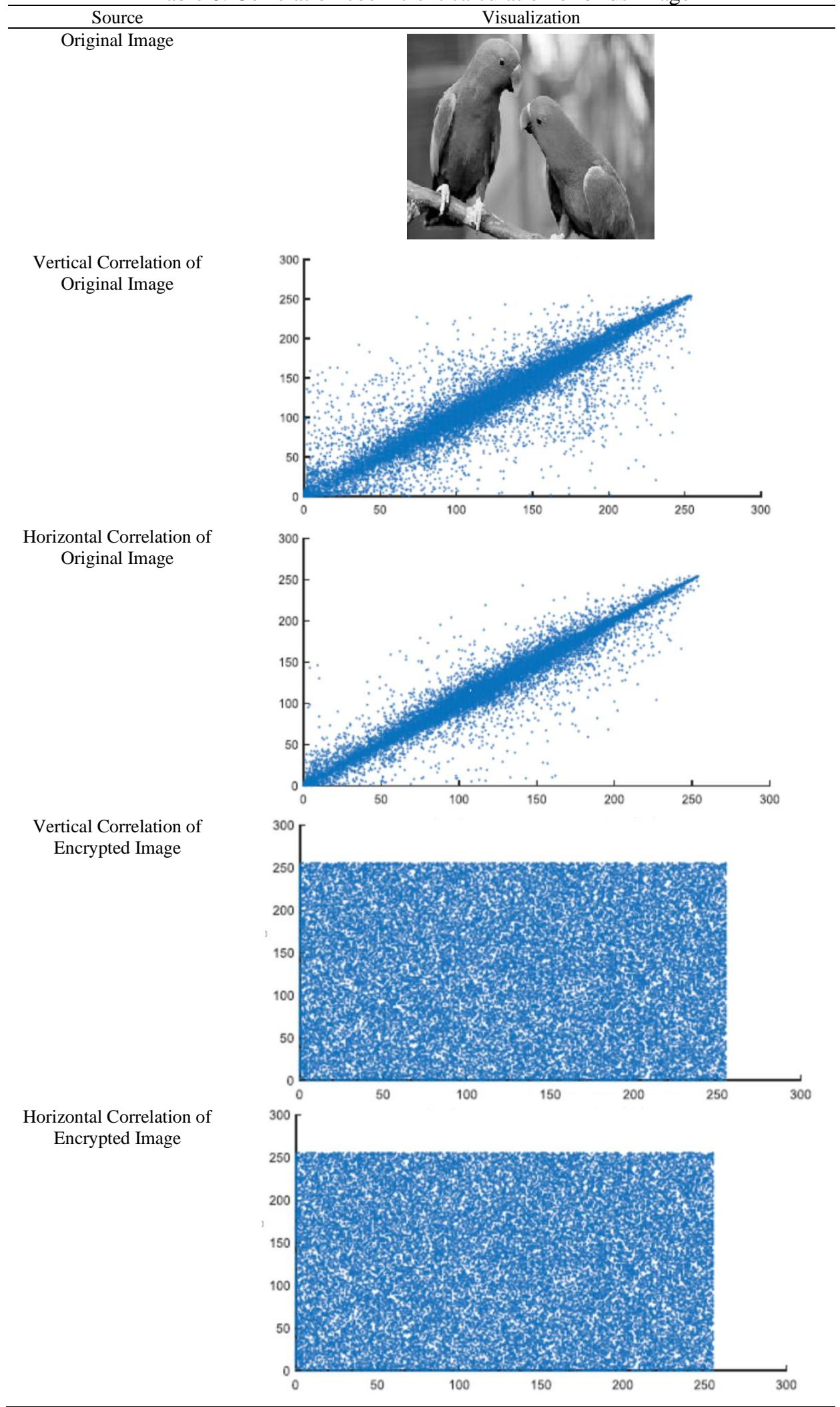


Table 9. Correlation coefficient calculation of butterflies image

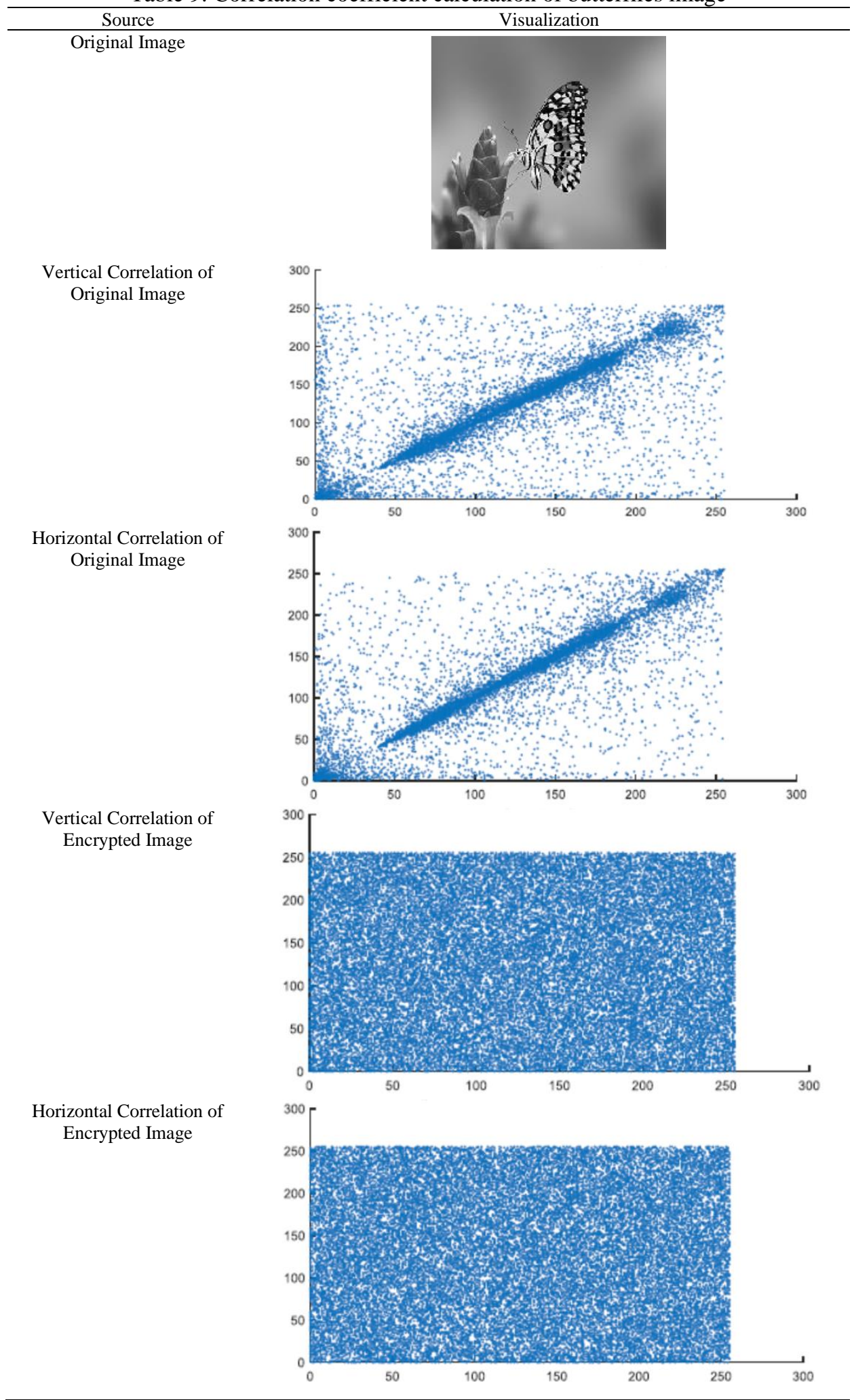

Table 10. The NPCR and UACI of owl image

\begin{tabular}{ccc}
\hline \multicolumn{3}{c}{ The NPCR and UACI Calculation } \\
\hline Measurements & Image & Calculation \\
NPCR & OWL & 99.7085 \\
UACI & OWL & 33.2030 \\
\hline
\end{tabular}


Table 11. Entropy analysis comparison

\begin{tabular}{lc}
\hline \multicolumn{1}{c}{ Method } & Entropy (Encryption Image) \\
\hline The Proposed Method 3D Chaos Map 3DCM & 7.9889 \\
Nested Piece Wise Linear Chaotic Map (NPWLCM) [22] & 7.9975 \\
Multiple Maps Combinations (MMC) [23] & 7.9997 \\
Linear Independence Scheme and the Logistic Map (LIS) and (LM) [24] & 7.9912 \\
Chaotic Encryption Algorithm (CEA) [25] & 7.9993 \\
Chaotic Coupled (CM) [26] & 7.9891 \\
mixture of chaotic maps (MCM) [27] & 7.9968 \\
Enhance 3D Chaotic MAP [28] & 7.9890 \\
\hline
\end{tabular}

Table 12. NPCR and UACI comparison

\begin{tabular}{lcc}
\hline \multicolumn{1}{c}{ Method } & NPCR $(\%)$ & UACI $(\%)$ \\
\hline The proposed method & 99.7085 & 33.2030 \\
NPWLCZ [22] & 99.6292 & 28.5050 \\
MMC [23] & 99.5100 & 33.4500 \\
CM and CF [29] & 99.6000 & 33.5400 \\
LIS and LM [24] & 99.5956 & 33.6035 \\
CEA [25] & 99.6128 & 33.4420 \\
CCML [30] & 25.0000 & 19.0000 \\
MCM [27] & 41.9620 & 33.2500 \\
3D Cat [31] (6 Round) & 50.3000 & 25.2000 \\
3D CBM [32] (5 Round) & 99.6000 & 33.4000 \\
Enhance 3D Chaotic MAP [28] & 99.6048 & 33.5044 \\
\hline
\end{tabular}

\section{CONCLUSION}

From the above results, the proposed method gave promising outcomes with the 3D chaotic graph for image encryption and decryption with perfect usages in real-time image cryptography. Three different images were implemented in this work and all gave robust results with the statistical analysis. The pixel diffusion method was implemented to change the position of the pixels of each image, then the entropy analysis was calculated. It is known that the coding and the visualization of the results were taken with MATLAB environment, so the entropy outcomes showed optimal results near to $7.89 \%$ with the encrypted owl image which is considered the ideal image in this work. All the proposed images were transformed from the 3D colored image to the 2D greyscale images before the operations were taken, the average calculation of the NPCR was $(99.7085 \%)$ and UACI was $(33.2030 \%)$. Finally, the keyspace gives higher space with $10^{\wedge} 124$ which made the proposed work powerful against different kinds of attacks.

\section{REFERENCES}

[1] D. R. Patel, "Information security: theory and practice," PHI Learning Pvt. Ltd., Apr.2008.

[2] C. P. Wu and C. C. J. Kuo, "Fast encryption methods for audiovisual data confidentiality," Multimedia Systems and Applications III, International Society for Optics and Photonics, vol. 4209, pp. 284-295, Mar. 2001, doi: 10.1117/12.420829.

[3] S. R. Maniyath and V. Thanikaiselvan, "An efficient image encryption using deep neural network and chaotic map," Microprocessors and Microsystems, vol. 77, pp. 103134, Sep. 2020, doi: 10.1016/j.micpro.2020.103134.

[4] M. I. Jordan and T. M. Mitchell, "Machine learning: Trends, perspectives, and prospects," Science, vol. 349, no. 6245, pp. 255260, Jul. 2015, doi: 10.1126/science.aaa8415.

[5] E. Hossain, I. Khan, F. Un-Noor, S. S. Sikander, and M. S. Sunny, "Application of big data and machine learning in smart grid, and associated security concerns: A review," IEEE Access vol. 24, no. 7, pp.13960-88, Jan. 2019, doi: 10.1109/ACCESS.2019.2894819.

[6] M. T. Gaata and F. F. Hantoosh, "An efficient image encryption technique using chaotic logistic map and rc4 stream cipher," International Journal of Modern Trends in Engineering and Research, vol. 3, no. 9, pp. 213-218, 2016, doi: 10.21884/IJMTER.2016.3068.YIAPY.

[7] G. R. Sinha and V. Bajaj, "Data deduplication applications in cognitive science and computer vision research", Data Deduplication Approaches: Concepts, Strategies, and Challenges, vol. 25, pp. 357, Nov. 2020, doi: 10.1016/B978-0-12-8233955.00001-X.

[8] S. Ahadpour, Y. Sadra, and Z. A. Arasteh Fard, "A Novel Chaotic Encryption Scheme based on Pseudorandom Bit Padding," International Journal of Computer Science Issues (IJCSI), vol. 9, no. 2, Jan. 2012

[9] M. I. Haider, A. Ali, D. Shah, and T. Shah, "Block cipher's nonlinear component design by elliptic curves: an image encryption application," Multimedia Tools and Applications, vol. 80, no. 3, pp. 4693-718, Jan. 2021, doi: 10.1007/s11042-020-09892-5.

[10] W. H. Chen, S. Luo, and W. X. Zheng, "Impulsive Synchronization of Reaction- Diffusion Neural Networks with Mixed Delays and Its Application to Image Encryption," in IEEE Transactions on Neural Networks and Learning Systems, vol. 27, no. 12, pp. 2696-2710, 2016, doi: 10.1109/TNNLS.2015.2512849.

[11] M. Dridi, M. A. Hajjaji, B. Bouallegue, and A. Mtibaa, "Cryptography of medical images based on a combination between chaotic and neural network," in IET Image Processing, vol. 10, no. 11, pp. 830-839, 2016, doi: 10.1049/iet-ipr.2015.0868.

[12] M. W. Fakhr, "A multi-key compressed sensing and machine learning privacy preserving computing scheme," 5th International Symposium on Computational and Business Intelligence (ISCBI), pp. 75-80, 2017, doi: 10.1109/ISCBI.2017.8053548. 
[13] R. G.-Bachrach, N. Dowlin, K. Laine, K. Lauter, M. Naehrig, and J. Wernsing, “Cryptonets: Applying neural networks to encrypted data with high throughput and accuracy," In International Conference on Machine Learning, pp. 201-210, 2016.

[14] F. Hu, J. Wang, X. Xu, C. Pu, and T. Peng. "Batch Image Encryption Using Generated Deep Features Based on Stacked Autoencoder Network," Mathematical Problems in Engineering, 2017, doi: 10.1155/2017/3675459.

[15] D. Hu, L. Wang, W. Jiang, S. Zheng and B. Li," A novel image steganography method via deep convolutional generative adversarial networks," IEEE Transactions on Information Forensics and Security, vol. 6, pp. 38303-38314, 2018, doi: 10.1109/ACCESS.2018.2852771.

[16] Q. Lu, C. Zhu, and X. Deng, "An efficient image encryption scheme based on the LSS chaotic map and single S-box," IEEE Access, vol. 8, pp. 25664-25678, 2020, doi: 10.1109/ACCESS.2020.2970806.

[17] A. Shifa et al., "Joint crypto-stego scheme for enhanced image protection with nearest-centroid clustering," IEEE Access, vol. 6, pp. 16189-16206, 2018, doi: 10.1109/ACCESS.2018.2815037.

[18] H. Liu, A. Kadir, and C. Xu, "Cryptanalysis and constructing S-Box based on chaotic map and backtracking," Applied Mathematics and Computation, vol. 376, pp. 125153, 2020, doi: 10.1016/j.amc.2020.125153.

[19] M. Rungruanganukul and T. Siriborvornratanakul, "Deep Learning Based Gesture Classification for Hand Physical Therapy Interactive Program, In: Duffy V. (eds) Digital Human Modeling and Applications in Health, Safety, Ergonomics and Risk Management," Posture, Motion and Health. HCII, Lecture Notes in Computer Science, vol 12198. Springer, Cham, 2020, doi: 10.1007/978-3-030-49904-4_26.

[20] P. N. Khade and M. Narnaware, "3D chaotic functions for image encryption," International Journal of Computer Science Issues (IJCSI), vol. 9, no. 3, pp.323, 2012.

[21] Z. Zhe, Y. Haibing, Z. Yu, P. Wenjie, and Z. Yunpeng, "A block encryption scheme based on 3D chaotic Arnold maps," In 2009 International Asia Symposium on Intelligent Interaction and Affective Computing, pp. 15-20, Dec. 2009, doi: 10.1109/ASIA.2009.47.

[22] H. H. Abdlrudha and Q. Nasir, "Low complexity high security image encryption based on nested PWLCM chaotic map," In 2011 International Conference for Internet Technology and Secured Transactions, ISBN 978-1-4577-0884-8, pp. 220-225, Dec. 2011.

[23] K. Faraoun, "Chaos-Based Key Stream Generator Based on Multiple Maps Combinations and its Application to Images Encryption," The International Arab Journal of Information Technology, vol. 7, no. 3, pp. 231-240, Jul. 2011.

[24] H. M. Al-Najjar, "Digital Image Encryption Algorithm Based on a Linear Independence Scheme and the Logistic Map," in Proceedings of ACIT, pp. 172-175, 2011.

[25] A. Awad and D. Awad, "Efficient Image Chaotic Encryption Algorithm with No Propagation Error," ETRI Journal, vol. 32, no. 5, pp 774-783, Oct. 2010, doi: 10.4218/etrij.10.1510.0063.

[26] M. Ahmad and M. S. Alam, "A New Algorithm of Encryption and Decryption of Images Using Chaotic Mapping," International Journal on Computer Science and Engineering, vol. 2 no. 1, pp. 46-50, 2009.

[27] S. Behnia, A. Akhshani, H. Mahmodi, and A. Akhavan, "A novel algorithm for image encryption based on mixture of chaoticmaps," ELSEVIER Chaos, Solitons \& Fractals, vol. 35, no. 2, pp. 408-419, Jan. 2008, doi: 10.1016/j.chaos.2006.05.011.

[28] M. B. Hossain, M. T. Rahman, A. B. M. S. Rahman, and S. Islam, "A new approach of image encryption using 3D chaotic map to enhance security of multimedia component," International Conference on Informatics, Electronics and Vision (ICIEV), 2014, doi: 10.1109/iciev.2014.6850856.

[29] A. Masmoudi, M. S. Bouhlel, and W. Puech, "A New Image Cryptosystem Based On Chaotic Map And Continued Fractions," 18th European Signal Processing Conference (EUSIPCO-2010), Aalborg, Denmark, pp. 1504-1508, Aug. 2010.

[30] S. Ahadpour and Y. Sadra, "A chaos-based image encryption scheme using chaotic coupled map lattices," International Journal of Computer Applications, vol. 49, no. 2, pp. 973-93-80869-31-6, 2012, doi: 10.5120/7599-0311.

[31] G. Y. Chen, Y. B. Mao, and C. K. Chui, "A symmetric image encryption scheme based on 3D chaotic cat maps," Chaos, Solitons and Fractals, vol. 21, no. 3, pp. 749-761, 2004, doi: 10.1016/j.chaos.2003.12.022.

[32] Y. Maa, G. Chen, and S. Lian, "A Novel Fast Image Encryption Scheme Based on 3D Chaotic Baker Maps," International Journal of Bifurcation and Chaos, vol. 14, no. 10, pp 3613-3624, 2004, doi: 10.1142/S021812740401151X.

\section{BIOGRAPHIES OF AUTHORS}

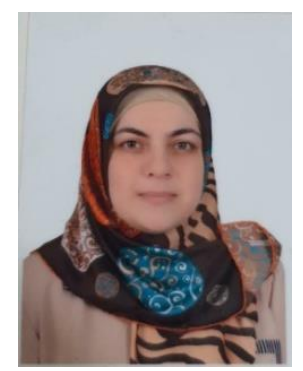

Daniah Abdul Qahar Shakir (iD SC SC works in Computer Science \& Information Technology where she has been a college employee since 2006. From 2007-2019, he taught many laboratory materials and programming language. Now she studies MSc degree at the University of Anbar, College of Computer and his undergraduate studies at the same University. Her current research include Image Processing, Artificial Intelligence and security methods. She can be contacted at email: dan14c1001@uoanbar.edu.iq.

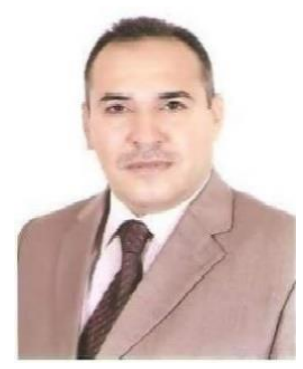

Ali Jbaeer Dawood (D) IS SC P has received Ph. D. in Computer science, Iraqi Commission for Computers and Informatics-Informatics Institute for Postgraduate Studies (2010). He joined in (2000-2003) to Computer Science Department College of Science-Baghdad University, Baghdad, Iraq as an external lecturer. He joined in (2001-2003) to Computer Science Department College of Computer, Anbar University, Anbar, Iraq as an external lecturer. He joined in (2002-2007) to Computer Science Department, AL-Mansour University College, Baghdad, Iraq as an external lecturer. He joined in (2005-2007) to Computer Science Department, College of Science. He can be contacted at email: co.dralijd@uoanbar.edu.iq. 